\title{
Henric Mirou (1551-1621) : Ein dichtender Apotheker im Streit der Konfessionen
}

\author{
Gamer, Maximilian
}

Posted at the Zurich Open Repository and Archive, University of Zurich ZORA URL: https://doi.org/10.5167/uzh-143404

Book Section

Published Version

Originally published at:

Gamer, Maximilian (2017). Henric Mirou (1551-1621) : Ein dichtender Apotheker im Streit der Konfessionen. In: Cardelle de Hartmann, Carmen; Eigler, Ulrich. Latein am Rhein : Zur Kulturtopographie und Literaturgeographie eines europäischen Stromes. Berlin/Boston: De Gruyter, 244-260. 
Sonderdruck aus:

\section{Latein am Rhein}

Zur Kulturtopographie und Literaturgeographie eines europäischen Stromes

Herausgegeben von

Carmen Cardelle de Hartmann und Ulrich Eigler unter Mitarbeit von Dörthe Führer und Brigitte Marti

\section{DE GRUYTER}


ISBN 978-3-11-040016-8

e-ISBN (PDF) 978-3-11-040028-1

e-ISBN (EPUB) 978-3-11-040032-8

ISSN 0934-5531

Library of Congress Cataloging-in-Publication Data

A CIP catalog record for this book has been applied for at the Library of Congress.

Bibliografische Information der Deutschen Nationalbibliothek

Die Deutsche Nationalbibliothek verzeichnet diese Publikation in der Deutschen Nationalbibliografie; detaillierte bibliografische Daten sind im Internet über http://dnb.dnb.de abrufbar.

(C) 2017 Walter de Gruyter GmbH, Berlin/Boston

Satz: fidus Publikations-Service $\mathrm{GmbH}$, Nördlingen

Druck und Bindung: Hubert \& Co. GmbH \& Co. KG, Göttingen

@ Gedruckt auf säurefreiem Papier

Printed in Germany

www.degruyter.com 


\section{Inhalt}

Ulrich Eigler

Einleitende Überlegungen — VII

\section{Geographie und Reise}

Martin Korenjak

Freigeboren. Die Ursprünge des Schweizer Rheins - 3

Katharina Suter-Meyer

Der Rhein: Fluss der Germanen oder der Helvetier? Patriotismus und Apologie in Vadians Kommentar zu Pomponius Mela (1522) — 22

Christoph Galle

hinc ditia Rheni flumina prospectas. Die Rheinreise des Erasmus von Rotterdam im Jahr 1514 und ihre literarischen Zeugnisse — 53

\section{Dichtung und Fluss}

\section{Beate Hintzen}

Ein Münsteraner in einer rheinischen Metropole. Bernardus Mollerus' Blick auf Köln -69

Seraina Plotke

Hydrographie als poetisches Prinzip der Digression. Die Flussbeschreibung Rhenus et eius descriptio elegans (1570) von Bernardus Mollerus — 82

Thomas Gärtner

Die frühen Werke des Münsteraners Bernhardus Mollerus: der Rhenus und die Ecclesias -94

Eckard Lefèvre

Der Rhein im Weltbild des Elsässers Jakob Balde — 106 
VI Inhalt

Stefan Tilg

Rheinromantik und Vater Rhein. Zwei Motive des deutschen

Humanismus -128

\section{Christian Guerra}

Basels sicherer Hafen: Inszenierung eines humanistischen Dialogs am Ufer des Rheins. Zu Enea Silvio Piccolominis Libellus dialogorum — 141

\section{Elisabeth Weber-Reber}

Trauer und Repräsentation. Der Rhein als poetische Landschaft in Basels neulateinischen Epicedien des 17. Jahrhunderts — 170

Henriette Harich-Schwarzbauer

Paul Schede (Paulus Melissus) - Rhene felix! Eine poetologische Lektüre 186

\section{Der Rhein als Achse einer res publica literaria}

Hans Schönemann

Wie ein Gelehrter reisen soll: Reiseregeln und Lob des Niederrheins in Georg Loys' Schrift Pervigilium Mercurii — 199

\section{Cristina Ricci}

Liceat ex illo felicissimo amne haurire, qui ex ore vere aureo velut ex fonte ditissimo promanat. Johannes Chrysostomus im Oberrheinischen Humanismus -220

\section{Maximilian Gamer}

Henric Mirou (1551-1621): Ein dichtender Apotheker im Streit der Konfessionen -244

Michael Hanstein

Regionale Identifikationsangebote im Straßburger Akademietheater. Zu den Dramen Julius Caesar und Moses des Caspar Brülow — 261

Namenregister $-\mathbf{2 8 3}$

Topographisches Register -287 


\section{Maximilian Gamer}

\section{Henric Mirou (1551-1621): Ein dichtender Apotheker im Streit der Konfessionen}

Der Tod Johann Casimirs, des Administrators der Kurpfalz, im Jahr 1592 traf die Intellektuellen in der Region schwer. Mehrheitlich reformiert eingestellt, erhofften sie sich nach den erst kurz zuvor überwundenen konfessionellen Turbulenzen mehr Zeit, um die reformierte Position vor Ort zu stabilisieren. Religion und Politik waren in der Kurpfalz mehr noch als anderorts in der Frühen Neuzeit eng verflochten. ${ }^{1}$ Diesen Komplex begleitete und illustrierte eine entsprechend konfessionspolitisch gefärbte Literaturproduktion. ${ }^{2}$ Umfangreiche Zeugnisse hierfür finden sich nicht nur in vom Hof sanktionierten, programmatisch-theologischen Werken wie etwa dem Heidelberger Katechismus, sondern auch in zahlreichen anderen Werken der Kurpfälzer Intellektuellen. Der besondere Stellenwert der konfessionellen Überzeugung im lateinischen Späthumanismus zeigt sich in einer Vielzahl von Dichtungen und Prosatexten, die entsprechende Themen direkt oder indirekt behandeln. In ihnen positionierte sich die gelehrte Öffentlichkeit der Kurpfalz und warb für Beibehaltung oder Wechsel der Politik und damit der konfessionellen Verfassung. ${ }^{3}$ Diese konfessionell-politische Verfas-

\footnotetext{
1 „Nirgendwo im Heiligen Römischen Reich Deutscher Nation gehören in der Frühen Neuzeit Religion und Politik so eng zusammen wie in der Kurpfalz. In kaum einem anderen deutschen Territorium war die Religion, genauer gesagt: die Konfession, in jener Zeit der wichtigste Bestandteil der Innen- und zeitweise der Außenpolitik, kaum irgendwo wurde die Politik weithin verstanden als Instrument der Religion.“ Eike Wolgast: Religion und Politik in der Kurpfalz im 17. Jahrhundert. In: Mannheimer Geschichtsblätter NF 6 (1999), S. 189-208, hier S. 189.

2 Dieser Beitrag baut auf einem von mir mitverfassten Artikel auf: Maximilian Gamer, Jörg Diefenbacher: Größer und berühmter als Aeneas: Johann Casimir. Zwei Gelegenheitsgedichte des Frankenthaler Apothekers Henric Mirou von 1592 an den kurpfälzischen Hof. In: Zeitschrift für die Geschichte des Oberrheins 160 (2012), S. 253-274. Des weiteren: Maximilian Gamer: Trauergedicht auf Johann Casimir und Glückwunschgedicht auf den Regierungsantritt Friedrichs IV. In: Die Wittelsbacher am Rhein. Die Kurpfalz und Europa. Hg. von Alfried Wieczorek u. a. Bd. 2. Kat. Nr. A3.08. Regensburg 2013 (Publikationen der Reiss-Engelhorn-Museen Mannheim 60), S. 95 f. $3 \mathrm{Zu}$ dieser Positionierung s. z. B. Wilhelm Kühlmann: Ein Heidelberger Dichter wünscht „prädestiniert“ zu sein. Zur Behandlung konfessionalistischer Positionen in der geistlichen Lyrik des deutschen Späthumanismus, ausgehend von einer Ode des Paul Schede Melissus (Meletemata I, 21) von 1595. In: Prädestination und Willensfreiheit. Luther, Erasmus, Calvin und ihre Wirkungsgeschichte. Festschrift für Theodor Mahlmann zum 75. Geburtstag. Hg. von Wilfried Härle, Barbara Mahlmann-Bauer. Leipzig 2009 (Marburger Theologische Studien 99), S. 146-158. Allge-
} 
sung war durchdrungen vom Gedankengut des Calvinismus, der mobilsten und, wenn man so will, internationalsten der protestantischen Bewegungen des sechzehnten Jahrhunderts. ${ }^{4}$ Geographisch wie auch intellektuell stellte die Kurpfalz unter den reformierten Kurfürsten der Linie Pfalz-Simmern gewissermaßen ein Zentrum für die reformierten Gemeinden in West- und Osteuropa dar. Der die Pfalz durchströmende Rhein bildete eine Verbindungslinie für Nachrichten und Ideen zwischen den Gemeinden der deutschsprachigen Schweiz und der spanischen Niederlande. ${ }^{5}$

Nach dem Tod von Ottheinrich von der Pfalz, der die lutherische Reformation in der Kurpfalz durchgesetzt hatte, ${ }^{6}$ im Jahr 1559, war die Herrschaft auf Friedrich III. und die Linie Pfalz-Simmern übergegangen. Da Friedrich zunehmend der calvinistischen Reformation anhing, intervenierte er - vor allem antikatholisch motiviert - in den spanischen Niederlanden und in Frankreich zugunsten der Hugenotten. Unter seiner Herrschaft wurden Klöster aufgehoben, Gebäude und Besitz teilweise an verfolgte Calvinisten übertragen. Die Pfalz wurde für diese zu einem sicheren Hafen, so dass ab 1562 eine Reihe von Exulantengemeinden auf aufgelöstem Klosterbesitz wie etwa in Schönau oder Frankenthal entstanden. ${ }^{7}$

meiner: Gerrit Walther: Humanismus und Konfession. In: Späthumanismus. Studien über das Ende einer kulturhistorischen Epoche. Hg. von Notker Hammerstein, Gerrit Walther. Göttingen 2000, S. 113-127.

4 Calvinism was the most dynamic and disruptive religious force of the later sixteenth century. Its emergence on the international scene shattered the precarious equilibrium established in the first generation of the Reformation, and precipitated three generations of religious warfare. Andrew Pettegree u. a.: Calvinism in Europe 1540-1620. Cambridge 1994, hier S. i.

5 Vgl. Hermann Wiegand: Der zweigipflige Musenberg. Die Literatur der Kurpfalz im Zeitalter des Humanismus. In: Der zweigipflige Musenberg. Studien zum Humanismus in der Kurpfalz. Hg. von Hermann Wiegand. Ubstadt-Weiher 2000 (Historische Schriften / Rhein-Neckar-Kreis 2), S.11-28, hier S.19-22; Dieter Mertens: Hofkultur in Heidelberg und Stuttgart um 1600. In: Hammerstein, Walther (Anm.3), S. 65-83. Ein Überblick über den politischen Hintergrund findet sich bei Eike Wolgast: Reformierte Konfession und Politik im 16. Jahrhundert. Studien zur Geschichte der Kurpfalz im Reformationszeitalter. Heidelberg 1998 (Schriften der Philosophischhistorischen Klasse der Heidelberger Akademie der Wissenschaften 10); Wilhelm Kreutz u.a. (Hgg.): Die Wittelsbacher und die Kurpfalz in der Neuzeit. Zwischen Reformation und Revolution. Regensburg 2013; Volker Press: Die „Zweite Reformation“ in der Kurpfalz. In: Die reformierte Konfessionalisierung in Deutschland - Das Problem der „Zweiten Reformation“. Hg. von Heinz Schilling. Gütersloh 1985 (Schriften des Vereins für Religionsgeschichte 195), S. 104-129. Kulturgeschichtliches bei: Karla Apperloo-Boersma, Herman J. Selderhuis (Hgg.): Macht des Glaubens. 450 Jahre Heidelberger Katechismus. Göttingen 2013.

6 Zum Beginn der Reformation in der Kurpfalz: Wolgast (Anm. 5), S. 17-32.

7 Vgl. Wolgast (Anm. 5), S. 33-73; Anton Schindling: Die reformierten Kurfürsten aus der Linie Pfalz-Simmern. In: Kreutz (Anm. 5), S. 14-43, hier S. 18-23. 


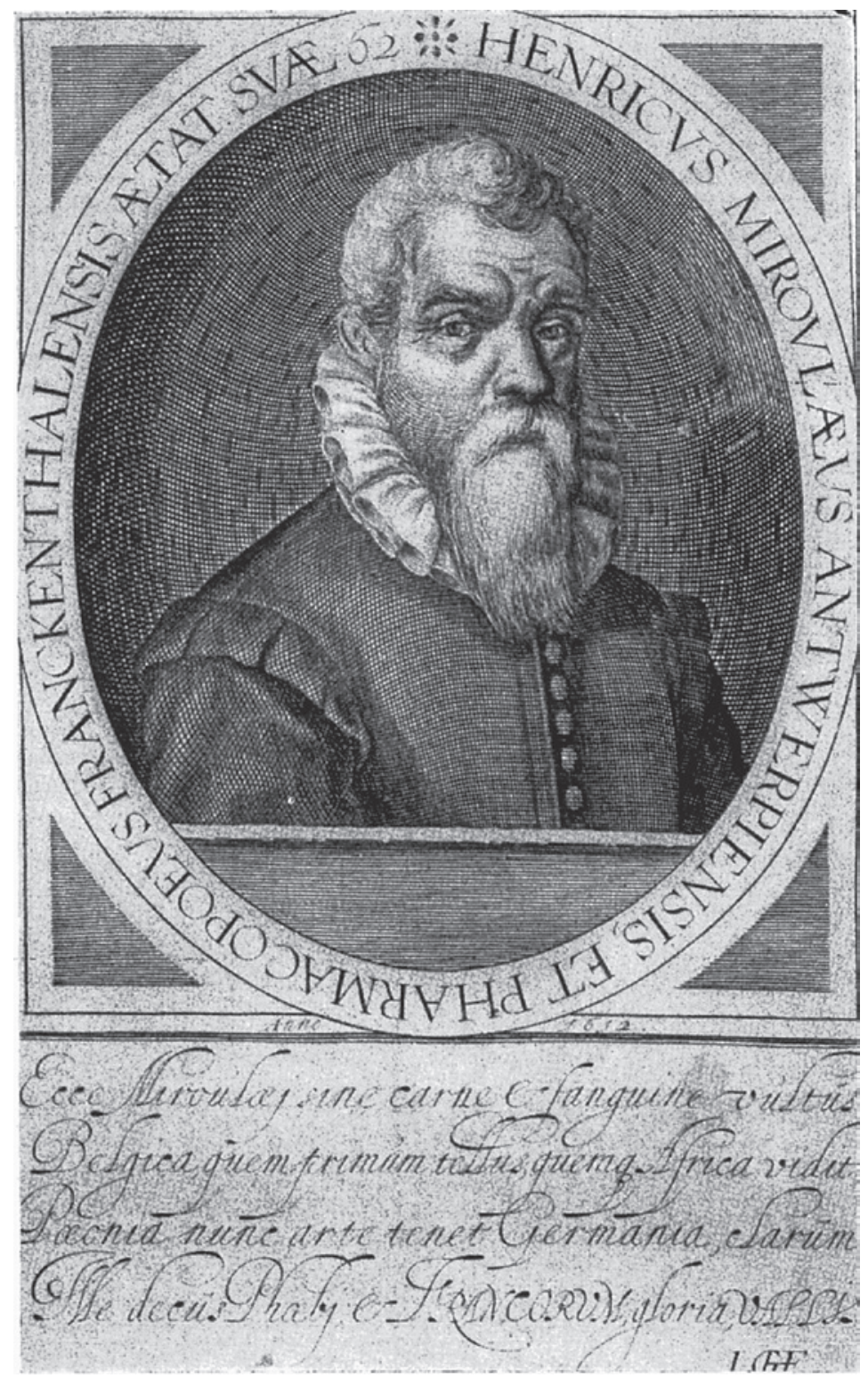

Abb.: Jacques Granthomme: Henric Mirou, 1612. Standort unbekannt. ${ }^{8}$

8 Reproduktion nach Jörg Diefenbacher: Anton Mirou 1578 - vor 1627. Ein Antwerpener Maler in Frankenthal. Landau 2007, S. 12. 
Das aus der Universität herausgelöste Heidelberger Collegium Sapientiae wie auch das Umfeld des Hofes entwickelten sich zu theologisch-intellektuellen Zentren. ${ }^{9}$ Bleibende Wirkung auf die reformierten Kirchen erzielte hier vor allem der 1563 verfasste Heidelberger Katechismus. Unterbrochen wurde diese Entwicklung durch die lutherische Zwischenphase unter Ludwig VI. (1567-1583), der bereits drei Monate nach dem Tod seines Vaters begann, das Land vom calvinistischen Einfluss zu säubern. ${ }^{10}$ Der Versuch, die Universität mit der Konkordienformel wieder auf lutherische Linie zu zwingen, führte zur Abwanderung vieler Gelehrter - eine Ausweichstelle wurde das Casimirianum in Neustadt, das als reformierte Hochschule Heidelberg zwischenzeitlich ersetzte. ${ }^{11}$ Johann Casimir, der in seinem Herrschaftsbereich als Pfalzgraf von Lautern die calvinistische Linie seines Vaters beibehalten hatte, führte nach dem Tod seines Bruders als Vormund für dessen Sohn schließlich die reformierte Konfession für die gesamte Kurpfalz wieder ein. ${ }^{12}$ Diese häufigen Richtungswechsel hinterließen Spuren in den Biographien und Werken der Kurpfälzer Autoren. Exemplarisch zeigt die Biographie des Paul Schede Melissus, des bekanntesten Vertreters der Wahl-Heidelberger, eindrucksvoll, wie die pro-calvinistische Politik die Kurpfalz zum überregionalen Anziehungspunkt für Gelehrte machte, wie diese unter dem Lutheraner Ludwig VI. die Pfalz verließen und nach der Wiedereinführung des Calvinismus durch Johann Casimir zurückkehrten. ${ }^{13}$

Allgemein sind der hohe Stellenwert der Konfession und die gemeinsame Basis griechisch-lateinischer Bildung, vielfach als Weg zum sozialen Aufstieg, Kennzeichen des deutschen Humanismus. Sie finden sich auch in der Biographie und dem Werk einer Randfigur der zweiten großen humanistischen Blütezeit

9 Vgl. Eike Wolgast: Das Collegium Sapientiae in Heidelberg im 16. Jahrhundert. In: Zeitschrift für die Geschichte des Oberrheins 147 (1999), S. 303-318.

10 Vgl. Wolgast (Anm. 5), S. 74-81; Schindling (Anm. 7), S. 23-28.

11 Vgl. Wolgast (Anm. 5), S. 76-78; Eike Wolgast: Die Statutenveränderungen der Universität Heidelberg zwischen 1558 und 1786. In: Kreutz (Anm. 5), S. 187-204, hier S. 195-198; Gustav Adolf Benrath: Das Casimirianum, die reformierte hohe Schule in Neustadt an der Haardt (1578-1584). In: Ruperto Carola. Heidelberger Universitätshefte 75 (1986), S. 31-37.

12 Vgl. Wolgast (Anm. 5), S. 82-90; Schindling (Anm. 7), S. 23-28; Paul Warmbrunn: Pfalz-Lautern. Grundzüge der Territorial-, Konfessions- und Geistesgeschichte. In: Kreutz (Anm. 5), S. 63-79.

13 Vgl. Eckart Schäfer: Paulus Melissus Schedius (1539-1602). Leben in Versen. In: Humanismus im deutschen Südwesten. Biographische Profile. Hg. von Paul Gerhard Schmidt. Sigmaringen 1993, S. 239-263. Weitere Literatur bei: Jörg Robert: Heidelberger Konstellationen um 1600. Paul Schede Melissus, Martin Opitz und die Anfänge der Deutschen Poeterey. In: Kreutz (Anm.5), S. 373-387, hier S. 375. „Mit Schede-Melissus strebt der lateinische Gipfel des zweigipfligen Musenbergs [Heidelberg] gegen Ende des sechzehnten Jahrhunderts einem nicht wieder erreichten Höhepunkt zu.“ Wiegand: Humanismus (Anm. 5), S. 21. 
Heidelbergs im Kreis um Schede Melissus: Henric Mirou (1551-1621), Apotheker und Gelegenheitsdichter der Exulantengemeinde in Frankenthal. Mirou, heute weitgehend unbekannt, kann exemplarisch den engen Bezug von Literatur und insbesondere Dichtung zur konfessionellen Politik aufzeigen. Daher beschäftigt sich dieser Beitrag, nach einer kurzen biographischen Einordnung und einem Überblick über Mirous erhaltenes Euvre, mit der konfessionellen Aussage der beiden Gedichte Mirous anlässlich des Todes Johann Casimirs und des Wechsels der Herrschaft auf Friedrich IV.

Mirou lebte spätestens ab 1586 als Apotheker in Frankenthal, wo er bis zu seinem Tod 1621 verblieb. Weiteres erfahren wir durch seinen Vermerk in Lotichius' Bibliotheca Poetica:

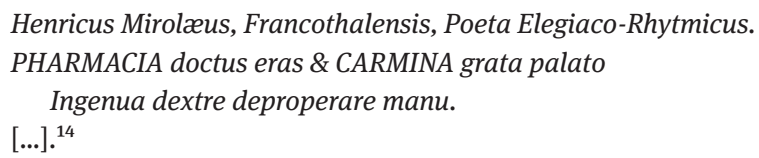

[Henri Mirou aus Frankenthal; elegisch-rhythmischer Dichter Du warst ausgebildet in der Arznei, und Gedichte, die wohltuend für den Gaumen sind, Eilten gewandt aus deiner freien Hand.]

In der Bibliotheca ist er wider Erwarten nicht im Kreis der Germaniae et Belgii Poetae des dritten Bandes, sondern im vierten Band der gefeierten Dichter verschiedener Nationes mit einigen Beispielen seines Wirkens aufgeführt. Mit sechs Seiten ist seine Biographie eine der umfangreicheren unter den 114 in der Bibliotheca behandelten neulateinischen Poeten, und sie lässt auf ein bewegtes Leben schließen. Lotichius zufolge wurde Mirou 1551 in Antwerpen geboren und studierte nach einer ersten Ausbildung in den Grundlagen u. a. in Bologna. Er nahm am Afrikafeldzug Karls V. teil und kehrte danach zunächst in die spanischen Niederlande zurück, emigrierte aber im Zuge einer weiteren Verschlechterung der Verhältnisse für Reformierte in die Kurpfalz nach Frankenthal.

Bei der Zuordnung des Afrikaabenteuers machte Lotichius allerdings einen Fehler. Aufgrund von Mirous Lebensdaten kommt für ihn erst die Kampagne des Don Juan de Austria im Jahr 1573, während der Herrschaft Philipps II. von Spanien, in Frage und nicht der Tunisfeldzug Kaiser Karls V. im Jahr 1535. Für Mirous Selbstbild jedenfalls war seine Teilnahme an der Expedition offenbar zentral. Sein Porträt, das er 1612 von dem für seine Radierungen bekannten Spezialisten Jacques Granthomme anfertigen ließ, ist mit einem entsprechenden Epi-

14 Johann Peter Lotichius: Bibliotheca poetica [...]. Bd. 4. Frankfurt 1628, S. 103-108. 
gramm unterschrieben. ${ }^{15}$ Vier Hexameter (nicht Distichen wie man sie von Mirou erwarten würde) fassen sein Leben zusammen:

\author{
Ecce Miroulæi sine carne \& sanguine vultus \\ Belgica quem primum tellus, quemque Africa vidit \\ Pæonia nunc arte tenet Germania clarum \\ Ille decus Phobi \& Francorum gloria vallis. ${ }^{16}$
}

[Siehe das Antlitz des Mirou ohne Fleisch und Blut, den die belgische Erde zuerst, den Afrika sah,

Germanien hält nun den durch seine Heilkunst berühmten.

Jener ist eine Zierde des Apollon und der Ruhm Frankenthals.]

Eine geordnete Ausgabe von Mirous Dichtung existiert nicht. Das heute erhaltene und bekannte Material verteilt sich auf drei Quellen:

1. Die Beispiele in Lotichius' Bibliotheca. Es handelt sich dabei um einige Verse an Johannes Lucas, der als Mirous Mäzen bezeichnet wird. Lotichius nennt Lucas als Quelle für seine Informationen über Mirou. Dazu kommt ein Trauergedicht auf den Tod des nach Hannover emigrierten Antwerpeners Paul Pelsius. ${ }^{17}$

2. Ein Druck, der von Frankenthaler Honoratioren anlässlich der Durchreise von Friedrich V. und Elisabeth Stuart 1613 herausgegeben wurde, mit vier Epigrammen Mirous, die jeweils einen der ersten vier Kurfürsten mit Namen Friedrich behandeln. ${ }^{18}$

3. Eine elfseitige Flugschrift mit zwei Gedichten: Threnodia in Illustrissimi Invictique Herois ac Ducis, Domini Ioannis Casimiri Comitis Palatini ad Rhenum Ducis Bavariæ, \&c. Tutoris et Administratoris Palatinatus Electoralis, Obitum Luctuosissimum [...] Item, Carmen Gratulatorium in Illustrissimi, Potentissimique Principis, Domini Friderici IIII. Comitis Palatini Rheni, S. Romani Imperii Archidapiferi \& Electoris, Ducis Bavariæ, \&c. Franckenthaliam ingredientis felix auspicium. ${ }^{19}$

15 Siehe Abbildung.

16 Ebenfalls abgedruckt bei Lotichius (Anm. 14), S. 108.

17 Vgl. Lotichius (Anm. 14), S. 106-108.

18 Kurtze und eigentliche Beschreibung alles dessen, Was dem eintritt des Durchleuchtigst. F. und H. H. Friedrich Churf. Pfalzg. bei Rhein. Hertzog in Beyern \&c. miet derselben C. Gn. Königlich Ehegemahl Fraw Elisabethen princessin zu groß Britannien, zu Frankenthal ahngestellt und gehalten worden. Frankenthal 1613, S. 55.

19 Das einzige bekannte erhaltene Exemplar ist heute im Besitz der Bayrischen Staatsbibliothek, Signatur: Res/P.o.lat. 168 q. 
Die Flugschrift ließ Mirou von dem Neustädter Drucker Harnisch anlässlich des Antrittsbesuchs Friedrichs IV. in Frankenthal im Jahr 1592 besorgen. Entsprechend sind darin ein Glückwunschgedicht an den nun amtierenden Kurfürsten und ein Trauergedicht auf dessen gerade verstorbenen Onkel Johann Casimir enthalten. Diese beiden Gedichte sind nicht nur formell der bedeutendste Teil von Mirous erhaltenem Euvre, sondern auch der inhaltlich interessanteste, da der Autor darin deutlich seine Position als reformierter Exulant vertritt. Auch können sie im Kontext einer ganzen Reihe vergleichbarer, politisch motivierter Trauerdichtungen auf Johann Casimir betrachtet werden. Der Tod des Administrators der Kurpfalz im Januar 1592 und der Wechsel der Herrschaft zu Friedrich IV. führten zu einer größeren Zahl von Drucklegungen in der Kur- und Oberpfalz. Ein Überblick, der keinen Anspruch auf Vollständigkeit erhebt, umfasst neben lateinischen Trauerreden, deutschsprachigen Trauerpredigten und „Beschreibungen des Abgangs“20 vor allem eine Vielzahl von lateinischen, aber auch einige grie-

20 Bereits Mertens (Anm. 6), S. 73 Anm. 24 verwies auf die im Vergleich große Menge an Drucklegungen zu Casimirs Tod. Die nachfolgend genannten Drucke befinden sich im Besitz der Universitätsbibliothek Heidelberg. Die Trauerreden: Quirinius Reuter: Oratio de Vita et Morte Illustrissimi ac Potentissimi Principis \& Domini Iohannis Casimiri Palatinati Rheni, Tutoris \& Electoralis Palatinatus Administratoris, Ducis Bavariæ Patris Patriae. Heidelberg 1592; Iacob Kimedoncius: Oratio Lugubris: Memoriæ Illustrissimi Principis et Domini Iohannis Casimiri, Comitis Palatini ad Renum, Ducis Bavariæ, \&c. Tutoris, \& Adiministratoris Electorii Palatinatus: Patris Patriæ, Ecclesiarum et Scholarum nutricii, in primis verò inclytæ Academiæ heidelbergensis Patroni ac mecoenatis benignissimi. Heidelberg 1592; Daniel Tossanus: Oratio Funebris Memoriae Illustrissimi et Fortissimi Principis Ioannis Casimiri Comitis Palatini ad Rhenum, Tutoris et Electorii Palatinatus Administratoris, Ducis Bavariæ, [...]. Heidelberg 1592. Die Predigten/Beschreibungen: Ungenannt, Melchior Anger: Exequiæ Casimirianæ. Beschreibung des tödtlichen Abgangs und Begrebnus / des Durchleuchtigsten / Hochgeborenen Fürsten und Herren / Herren Johanns Casimiri / Pfalzgraffen bey Rhein / Vormunden und Administratorn der Churfürstlichen Pfalz / Herzogen in Bayern / \&c. Christlicher gedechtnuß. Sampt angehengter Leichpredig Melchioris Angeri Hoffpredigers / gehalten in der Churfürstlichen Stadt Heydelberg / im Stifft zum H. Geist / den 26. Januarii / dieses lauffenden 1592. Jars / als die Fürstliche Leych daselbst zu der Erden bestattet worden. [...] Heidelberg 1592; Balthasar Copius: Siehe auff Gott. Ein Predigt / Gehalten zu Newstadt an der Hardt / den 26. Januarii im Jahr 92. Als die Leich deß Durchleuchtigsten Hochgebornen Fürsten und Herrn / Herrn Johann Casimirn / Pfalzgraffen bey Rhein / und Herzogen in Bayern / \&c. Gewesenen Vormundt / und der Churfürstlichen Pfalz Administratoren, Unsers Gnädigsten Fürsten und Herrn / und frommen und getrewen Pflegers und Lehrers der Kirchen Christi / und rechten Vaters des Vaterlandes / hochseligster Christmiltester Gedächtenuß / zu Heydelberg in der Kirchen zum H. Geist mit grossem trawren und klagen ist zur Erden bestattet worden. Neustadt an der Hardt 1592; Georg Lupichius: Eine Klagpredigt Über den Tödtlichen Abgang / weiland des Durchleuchtigsten Hochgebornen Fürsten und Herrn / Herrn Johann Casimirs / Pfalzgrafens bey Rheyn / Vormunds und der Churfürstlichen Pfaltz Administratoris, Hertzogen in Bayrn / \&c. Christseliger gedechtnus / So den 6. Januarii dises 92. jars / umb 6. 
chische Trauer- und Glückwunschgedichte. Diese thematisch identischen, formal und inhaltlich nahestehenden Werke bieten sich zur vergleichenden Lektüre an, um die beiden politischen Dichtungen Mirous genauer einzuordnen und $\mathrm{zu}$ bewerten. Sie finden sich in den folgenden Sammlungen oder Einzeldrucken:

- Parentalia in Obitum Iohannis Casimiri, Comitis Palatini ad Rhenum, Ducis Boiorum, Administratoris Archipal. Principis et Fortissimi et Optimi: Qui decessit Haidelbergæ in Arce. Die VI Ian. Antiqui. von Paul Schede Melissus, Marquard Freher, Johannes Posthius und weitere Heidelberger. ${ }^{21}$

- Carmina Funebria In Obitum Luctuosissimum Ilustrissimi Fortissimi et nunquam satis Laudati Principis et Herois, Iohannis Casimiri, Comitis Palatini ad Rhenum, Ducis Bavariæ, \&c. Tutoris et Electorii Palatinatus Administratoris Fidelissimi, domini nostri clementissimi, ac de Republica et Ecclesia Orthodoxa eximie atque præclare: de Scholis Palatinatus plusquam Paterne promeriti. Darin vertreten sind die geistigen Honoratioren Neustadts. ${ }^{22}$

Als jeweils eigene Ausgaben existieren:

- Lacrimae in obitum præmaturum, funus luctuosissimum, memoriam sempiternam, honorem meritissimum Iohannis Casimiri [...] von Lambert Ludolf Helm. ${ }^{23}$

uhr gegen tag / zu Heydelberg im Schloß seliglich in Gott entschlaffen. Gehalten zu Amberg in S. Martins Kirchen. Durch Georgium Lupichium. Sampt / einem Deutschen Klaglied/ und etlichen Lateinischen Epitaphiis. Amberg 1592; Iohannes Strackius: Eine Christliche Leichpredigt Über dem Tödlichen abgang weiland des Durchleuchtigsten und Hochgeborenen Fürsten und Herren / Herrn Iohan Casimirs, der Churfürstlichen Pfalz Administratorn unnd Vormund / Pfalzgrafen beim Rein / und Herzogen in Baiern / welches F. G. in der Churfürstlichen Stat Heidelberg den 6 Januarii / Anno 1592, des morgens zwischen fünff und 6 uhren im Herrn seelig entschlaffen. Gehalten zu Cassel im Fürstenthum Hessen / in der Pfarkirchen auff der Freiheit / den 18 Januarii. [...] Heidelberg 1592; Ungenannt: Ware Beschreibung / Von dem tödtlichen Abgang und Begrebnuß / des Durchleuchtigsten Hochgeborenen Fürsten und Herrn / Herrn Johann Casimiri / Pfalzgraffen bey Rhein / Vormündern und der Churfürstlichen Pfalz Administratoris / Herzogen in Beyern / etc. Geschehen in der Churfürstlichen Stadt Heydelberg / in der Stifftskirche zum heiligen Geist / den 26. Januaris / Anno 1592. Sampt erzehlung irer F. G. Kranckheit und Trostsprüchen / so. I. F. G. biß am letzten Ende gebraucht habe / auch was vornemen Fürsten / Herrn und Adelspersonen erschienen / und Legaten / so ire F. Leyche begleitet haben / Beneben des Texts / so bey irer Fürstl: Leych gehalten worden. [Mit illustrierenden Kupferstichen]. Heidelberg 1592.

21 Parentalia [...]. O. O. 1592. Melissus gab bereits 1588 einen Druck mit Gedichten an Casimir und Friedrich IV. heraus: Paulus Melissus-Schede: Odæ Palatinæ. Ad serenissimos illustrissimosque Principes Ioannem Casimirum Administratorem, et Fridericum IV. [...]. Heidelberg 1588. 22 Carmina Funebria [...]. Neustadt an der Hardt 1592.

23 Lambert Ludolf Helm: Lacrimae [...]. Heidelberg 1592. 
- Iusta Funebria in Obitum Illustrissimi ac Fortissimi Memoriæ Sanctissimæ Principis ac Domini, Domini Iohannes Casimiri [...] von Johann Kahl. ${ }^{24}$

- Memoriae Illustrissimi herois Domini Iohannis Casimiri [...] \& Honori Florentissimi principis Domini Friderici Quarti [...] von Georg Remus, als Besonderheit weitgehend zweisprachig griechisch/lateinisch gehalten. ${ }^{25}$

Eine Sammelausgabe der meisten dieser und weiterer Trauergedichte erschien noch im gleichen Jahr. ${ }^{26}$ Die Autoren eint ihre pro-reformierte Haltung, auch wenn diese in unterschiedlichem Maße sichtbar wird. ${ }^{27}$ Da sie thematisch und formell gebunden waren, verwendeten die Autoren dieser Dichtungen jeweils ähnliche Motive und die Gedichte weisen folglich innerhalb des Korpus viele Parallelen auf. Mirou steht etwas außerhalb dieser Gruppe durch die anlassbedingt spätere Veröffentlichung Mitte des Jahres 1592, für einen Besuch Friedrichs IV. in Frankenthal.

Auch Mirou beginnt zunächst mit einem Trauergedicht. Seine Threnodia besteht aus 30 elegischen Distichen, die (ungewöhnlich für die Trauerdichtung für Casimir) ein doppeltes Akrostichon bilden, und zwar Namen und Titel des Beklagten Ioannes Casimirus comes palatinus, administrator palatinatus electo[r].

Das Gedicht ist chronologisch aufgebaut von den Vorzeichen für den Tod, über diesen selbst mit dem Trauerzug vom Heidelberger Schloss zur Heiliggeistkirche bis zu einer Würdigung der Lebensleistung des Verstorbenen. Auffällig ist mehr die wenig verklausulierte politisch-konfessionelle Positionierung des Autors. Auch wenn der Konflikt zwischen Lutheranern und Reformierten, der die Kurpfalz knapp zehn Jahre zuvor beschäftigt hatte, wie im Folgenden gezeigt nur vage angedeutet wird, steht am Ende der Threnodia explizit der Wunsch nach einem reibungslosen Übergang der Herrschaft auf Friedrich IV. ohne eine weitere Administratur eines möglicherweise pro-lutherischen Vormunds. Diese Hoffnung klingt auch in den Trauerdichtungen anderer Autoren an. ${ }^{28}$ Die konfessionelle

24 Johann Kahl: Iusta funebria [...]. Heidelberg 1592.

25 Georg Remus: Memoriae [...]. O. O. 1592.

26 Memoriæ nunquam satis laudatæ Christiani Saxoniæ Ducis et Iohannis Casimiri Comitis Palatini Rheni [...]. O. O. 1592.

27 Dies ist wenig verwunderlich, da viele der Autoren ein öffentliches Amt innehatten. Tatsächlich war der Rückhalt für die reformierte Politik in der Kurpfalz weitgehend auf die Intellektuellen beschränkt, während die weite Bevölkerung mehr lutherisch orientiert war. Vgl. Wolgast (Anm. 5), S. 86.

28 Vgl. Dieter Cunz: Die Regentschaft des Pfalzgrafen Johann Casimir in der Kurpfalz 1583-1592. Limburg an der Lahn 1934, S. 100 f. Als Beispiel dieser Position in der Dichtung bietet sich Melissus-Schede an, siehe hier Anm. 36, 37. 
Positionierung innerhalb des Gedichts findet auf zwei Ebenen statt: Im lobenden Nachruf auf den Verstorbenen sowie in einer Ansprache an Friedrich IV. Mirou stellt die Kriegszüge Casimirs direkt in den konfessionellen Bezug. Er lobt die aktive, also militärische Vorgehensweise Casimirs, sei es im Auftrag seines Vaters oder in eigener Sache.

\section{Interit Aeneas nulli pietate secundus \\ Tum, cùm humeris ferret sacra, patremque suis. \\ Maior at ille fide pietateque clarior olim \\ Obtulit hostili cum patre sacra gregi. ${ }^{29}$}

[Aeneas, der niemand an Pflichtbewusstsein nachstand, ging damals verloren, als er die Hausgötter und seinen Vater auf den Schultern trug. Dieser aber [Casimir], größer an Glaube und Pflichtbewusstsein, hat einst mit dem Vater die Heiligtümer vor der feindlichen Horde bewahrt.]

Auch in den anderen Nachrufen auf Casimir werden dessen militärischen Leistungen hervorgehoben, ${ }^{30}$ allerdings ohne diese dabei so direkt mit der Konfessionspolitik zu verknüpfen wie bei Mirou. Zudem stellt dieser die kriegerischheroischen Taten, entsprechend dem Selbstverständnis der in Heidelberg residierenden Wittelsbacher, ${ }^{31}$ in einen mythologischen Kontext Der Betrauerte habe Herakles und Hektor geglichen, ${ }^{32}$ an Tugend sogar Aeneas übertroffen. Umgesetzt ist dies in der Antithese von ferre / offerre: Während Aeneas seinen Vater und die Penaten aus dem brennenden Troja lediglich in Sicherheit trug (Verg. Aen. 2,717f.), leistete Casimir nicht nur erfolgreich Widerstand, sondern

29 Mirou: Threnodia (Anm. 19), S. 4. Ndr.: Gamer (Anm. 3), S. 260, V. $21-24$.

30 Besonders elegant gelöst ist dies bei Lambert Helm (Anm. 23): Ingens bellicrepis tu metus hostibus, / Tu, tu terror eras; Matrona, Arar, Liger, / Renus tergemino gurgite spumeum / Mergens Occeano caput, / Forti bella gerentem te animo \& manu, / Viderunt grege pro, legeque patria, / Hostem fœdifragum aduersús \& impium, / Supremi auxilio Dei; S. 4 f. Helm gibt mit den Flussnamen sogar die Regionen der einzelnen Kampagnen an, anstatt nur formelhaft auf die Kriegszüge Casimirs zu verweisen, wie dies die anderen Autoren handhaben, beispielsweise im Carmen [...] luctuosum obitum in den Carmina Funebria (Anm.22): In bellis quatuor potuit nec lædere Mavors. In heutiger Bewertung gilt Casimir als erfolgloser Feldherr: „Damit zeigte sich schon früh ein weiterer prägender Wesenszug [Casimirs]: seine Vorliebe für das Kriegshandwerk. In seiner Persönlichkeit war er von den Sitten des Feldlagers geprägt. Freilich stand dieser Feldzug - wie auch alle weiteren militärischen Unternehmungen Johann Casimirs - unter keinem glücklichen Stern. “ Warmbrunn (Anm.12), S. 66.

31 Stein geworden im Bildprogramm des Ottheinrich-Baus im Heidelberger Schloss mit seinen allegorischen, mythologischen und biblischen Heldenfiguren, s. Apperloo-Boersma, Selderhuis (Anm. 5), S. 358-364.

32 Vgl. Mirou: Threnodia (Anm. 19), S. 3. Ndr.: Gamer (Anm. 2), S. 260, V. 19. 
trägt den Glauben dem Feind entgegen. Zusätzlich bietet Mirou dieses Bild von Casimir und seinem Vater für seine politische Inhaltsebene die Möglichkeit, einen Bezug zu Friedrich III., dem Großvater Friedrichs IV., herzustellen. Dieser Verweis ist durchaus wichtig, da Casimir außerhalb der Erbfolge stand und daher nur bedingt als legitimierendes Vorbild für den jungen Friedrich dienen konnte. Das Gewicht des gerade für die Exulanten so bedeutsamen Großvaters als Vorbild baut Mirou in seinem Gedicht dabei weiter aus. Gilt das Lob zunächst nur dem verstorbenen Casimir, zielt es in den oben zitierten Versen bereits auf Casimir und seinen Vater zugleich. Nachdem Mirou zu einer direkten Anrede an den jungen Friedrich gewechselt hat, stellt er diesen unmittelbar in die Nachfolge seines gleichnamigen Vorgängers. Das Vorbild Casimirs übergeht er zwar nicht direkt, sein Schwerpunkt liegt aber tatsächlich mehr auf einer Kontinuität zwischen dem Großvater und dem Enkel. Der Großvater war es, der den reformierten Glauben in der Kurpfalz etablierte:

Maximus interiit fidei defensor auitæ, Impiger Ausonii dogmatis hostis obit.

Exulibus spes certa viris Casimirus, \& (eheu) Nunc iacet auxilium, præsidiumque scholæ.

Sanctaque (defuncto Propugnatore fideli, Acrior hoc, nullus cùm superesset, erat)

PRINCIPIS exequias Ecclesia cantibus orna, Terra tegat cineres. [.... $]^{33}$

[Der größte Verteidiger des großväterlichen Glaubens ist dahingegangen, der unermüdliche Feind der römischen Lehre ist tot. Casimir, die sichere Hoffnung und Hilfe für die verbannten Männer, ach, und Stütze der Schule liegt nun darnieder. Auch du, heilige Kirche, (beharrlicher als dieser gestorbene Vorkämpfer des Glaubens war zu seinen Lebzeiten niemand) [...].]

Mirou zeigt sich hier offen antikatholisch. Die primäre Bedrohung für die Reformierten sieht er in den gegenreformatorischen Bestrebungen. Nur indirekt nimmt er dagegen die inner-protestantischen Spannungen auf. Der Schutz für die Exulanten und die Stütze der (reformierten) Schule, die Casimir gewährte, bewahrten diese immerhin keineswegs vor katholischen Übergriffen, sondern vor der lutherischen Politik Ludwigs VI. ${ }^{34}$ Nicht zuletzt die abschließende direkte Anrede

33 Mirou: Threnodia (Anm. 19), S. 4. Ndr.: Gamer (Anm. 2), S. 260 f., V. 37-42.

34 Die Lutheranisierung unter Ludwig VI. verursachte für die rechtsrheinischen Exulantensiedlungen in Schönau und Heidelberg große Probleme. Vgl. Gerhard Kaller: Die Anfänge der kurpfälzischen Exulantengemeinden Frankenthal, Schönau, Heidelberg und Otterberg (1562-1590). 
Mirous an Friedrich IV. ist von einer aggressiven konfessionellen Polemik gekennzeichnet.

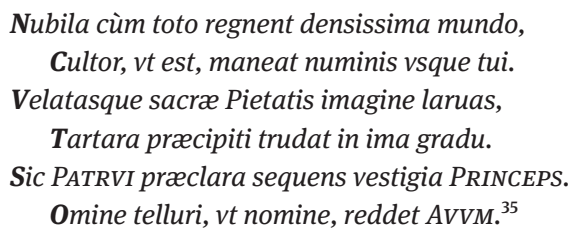

[Wenn auch auf der ganzen Welt die dichtesten Wolken herrschen, soll er unverwandt, wie er es jetzt schon ist, der Verehrer deines göttlichen Willens bleiben. Die hinter dem Schein der heiligen Frömmigkeit verschleierten Masken soll er mit entschlossenem Schritt in die tiefste Unterwelt drängen. Indem der Fürst so den berühmten Spuren des Onkels folgt, wird er der Welt im Wirken wie schon im Namen den Großvater zurückgeben.]

Wie oben bemerkt, ist der Wechsel der Herrschaft zu Friedrich IV. ohne eine weitere Administratur ein wesentliches Anliegen der Reformierten in der Pfalz. ${ }^{36}$ Dieser Wunsch zeigt sich in der betonten Übertragung der Herrschaft auf Friedrich selbst, wie auch in dem wiederkehrenden Motiv einer Kontinuität der vier Kurfürsten mit Namen Friedrich in der hier betrachteten kurpfälzischen Dichtung. ${ }^{37}$ Der ungeliebte Lutheraner Ludwig VI., Vater des Adressaten und Bruder des Betrauerten, bleibt in der Regel unsichtbar und bei Mirou hinter den dichten Wolken des Irrglaubens verborgen.

Während die politischen Wünsche des Dichters in der Threnodia auf die gesamte Kurpfalz und wohl auch auf eine intervenierend pro-reformierte Politik

In: Zeitschrift für die Geschichte des Oberrheins 147 (1999), S. 393-403, hier S. 397-399. Der sichere Hafen der Schule im Casimirianum war für die Intellektuellen der Pfalz allgemein ein wichtiger Punkt. So widmete Johannes Posthius ihm ein eigenes Epigramm, De Casimirano collegio, in den Parentalia (Anm. 21), S. 24. Ndr.: Wilhelm Kühlmann, Hermann Wiegand: Parnassus Palatinus. Humanistische Dichtung in Heidelberg und der alten Kurpfalz. Heidelberg 1983, S. 78 f. Insgesamt nimmt Mirou hier nur sehr zurückhaltend Stellung im inner-protestantischen Streit der Kurpfalz in dem wesentlich schärfer geschrieben wurde. Gipfel dürfte hier das nicht zugeordnete Distichon O Casimire potens, servos expelle Lutheri:/ Ense, rota, ponto, funibus, igne neca! sein, dass sich an verschiedenen Stellen überliefert findet. Hier zitiert nach: Gustav Benrath: Irenik und Zweite Reformation. In: Schilling (Anm. 5), S. 356.

35 Mirou: Threnodia (Anm. 19), S. 4. Ndr.: Gamer (Anm. 2), S. 261, V. 55-60.

36 Daher geschah beispielsweise für Melissus der Übergang der Herrschaft auf Friedrich IV. Raro iehovæ consilio Dei, Parentalia (Anm. 21) S. 13.

37 Vgl. Melissus' Ad Fridericum IV. Septemvirum Palatinum, Parentalia (Anm. 21), S. 38. Hier wünscht er sich, dass der junge Friedrich von jedem seiner gleichnamigen Vorfahren ein Attribut, Victoria, Sapientia und Pietas, übernehme. 
bezogen sind, ist die Stoßrichtung des auf sie folgenden Carmen Gratulatorium einem rein lokalen Interesse geschuldet: der herrschaftlichen Förderung der Stadt Frankenthal (und natürlich auch des Dichters selbst). Das Gedicht widmet sich dem Empfang Friedrichs IV. in Frankenthal und verbindet die an den Herrscher gerichteten Wünsche mit einem Enkomium auf Mirous gewählte Heimatstadt. Wieder wird der nahtlose Übergang der Herrschaft auf Friedrich IV. betont und seine Stellung als comes Palatinus ad Rhenum hervorgehoben. Der Rhein selbst soll ihm seine Aufwartung machen:

Rhene pater, tuque, ô, contermine Neccare Rheno

Tollite arundineis tempora operta comis.

PRINCIPIS aduentum cursu celebrate retento, Applaudatque suo squamea turba Dvcr. ${ }^{38}$

[Du, Vater Rhein, und du, oh Neckar, Nachbar des Rheines, erhebt eure schilfbekrönten Häupter. Preist die Ankunft des Fürsten durch verminderten Lauf, und euer schuppiges Gefolge soll seinem Fürsten applaudieren.]

Rhein und Neckar prägen die Geographie der Kurpfalz und stehen hier als Personifikation für diese. Das Motiv des ,Vater Rhein` ist gerade bei Mirous erklärtem Vorbild Melissus zu finden. ${ }^{39}$ Mirou ordnet dem Rhein als Gratulant ersten Ranges eine ganze Reihe von weiteren, über das Reichsgebiet verteilten Flüssen unter (auch solche die nicht Flusssystem des Rheins gehören), die ihre Wasser mit ihm vermischen und dem Fürst ihre Aufwartung machen sollen. ${ }^{40}$ Die Flussnamen mögen nun der metrischen Notwendigkeit geschuldet sein oder dem Bild des Vater Rhein als erstem unter den Flüssen im Reich, so wie der Pfalzgraf bei Rhein der bedeutendste unter den weltlichen Herrschern des Reichs ist.

Ein wesentliches Element des Gedichts ist auch die Stadtbeschreibung, welche von Mirou wiederum zur konfessionellen Positionierung eingesetzt wurde. Die rasch fortschreitende Entwicklung einer Exulantenkolonie $\mathrm{zu}$ einer wirtschaft-

38 Mirou: Threnodia (Anm. 19), S. 8. Ndr.: Gamer (Anm. 2), S. 267, V. 85-88.

39 S. Melissus' Ad Rhenum Flumen Germaniæ, in: Paulus Schede-Melissus: Schediasmata Poetica. Paris 1586, S. 229-231. Ndr.: Kühlmann, Wiegand (Anm. 34), S. 82 f. In der Verbindung beider Flüsse trauern Rhein und Neckar zusammen um Casimir in Schedes erster Nænia (Anm. 21), S. 3: Naiades ingeminant planctu suspiria, \& altos / Rhenus ab amne tacit, ceu Nicer ipse, sonos. Ähnlich bereits in der ebenfalls an Friedrich IV. adressierten Naenia anlässlich des Todes seines Vaters, des ungeliebten Lutheraners Ludwig VI.: Prolixiorum fimbria, verrens humum. / Occulit Nicer caput, / Caput occulit et Rhenus, / Amnicolaeque Deae. Sed ista te manent, / O puer invicte, incommoda. Paulus Melissus-Schede: Naenia in funere illustrissimi Principis Ludovici Comitis Palatini ad Rhenum, [...]. Nürnberg 1583, S. 6.

40 Vgl. Mirou: Threnodia (Anm. 19), S. 8. Ndr.: Gamer (Anm. 2), S. 267, V. 89-92. 
lich prosperierenden Stadt rief bei Zeitgenossen und Reisenden Bewunderung hervor. Ein Beleg hierfür ist die bereits Ende 1578 von Daniel Rogers verfasste Elegie Ad Francdaliam Coloniam Belgicam, in Palatinatu. ${ }^{41}$ Mirou präsentiert in 59 Versen knapp sein als libera vallis bezeichnetes Frankenthal. Zunächst schildert er die Entstehungsgeschichte des Ortes als Exulantenansiedlung, die den vernachlässigten Besitzungen des Augustinerstiftes Zivilisation bringt, dann hebt er die wirtschaftliche Bedeutung der Stadt für die Herrschaft hervor und bittet um weitere Förderung. Dabei betont er die Rechtgläubigkeit und Rechtschaffenheit der Bewohner - es gilt ihm darzustellen, dass die Besiedlung durch Flüchtlinge kein Makel für die Stadt ist, sondern ihr zur Zierde gereicht. ${ }^{42}$ Ein literarisches Vorbild und die Vorlage für seine Stadtbeschreibung fand Mirou wiederum in der Aeneis: Die durch die Verbrechen des Pygmalion vertriebene Tyrer um Dido erbauen ihren Zufluchtsort Karthago - der Anblick, der sich Aeneas bietet, wird im ersten Buch, Verse 419-436 geschildert. Mirou nahm aus dieser Passage Gliederung und Vokabular, soweit sie den Verhältnissen angemessen waren, und fand hier weiteren Raum für anti-katholische Polemik, insbesondere im Kontext der Umwidmung des ehemaligen Stiftes.

\footnotetext{
Exiguas ne sperne domos, nou mænia nobis Cernis ab exiguis condita principiis.

Antè cucullatis habita fuere cinædis Hæc loca, \& impuro contaminata grege

Sed fraudes niuea, cernens sub veste latentes, Et scelerata tuus facta perosus AVVS.

Diuinum reparans cultum, sit copia fandi Pauca, pecus veteri sede remouit iners. Interea patriis eiectos finibus, oris Appulit his Papæ sæua procella, viros. Omnibus exhaustis iam casibus, omnium egenis Non scelus exilii causa, nefasue fuit,
}

41 Zu Rogers s. Jan A. Dorsten: Poets, patrons, and Professors. Sir Philip Sidney, Daniel Rogers,
and the Leiden Humanists. Leiden 1962 (Publications of the Sir Thomas Brown Institute. General
Series 2), S. 9-75. Die genannte Elegie ist nur handschriftlich überliefert: Huntington Library, HM
31188, Bl. 39v-42v. Auszugsweise übersetzt mit Abdruck des vollständigen lateinischen Textes
bei: Thea Vignau-Wilberg: Dichter, Denker, Diplomaten. Daniel Rogers' Ode auf Frankenthal aus
dem Jahr 1578. In: Kunst, Kommerz, Glaubenskampf. Frankenthal um 1600. Hg. von Edgar J.
Hürkey, Ingrid Bürgy-de Ruijter. Worms 1995, S.48-52. 42 Dies ist eine deutliche Zuspitzung gegenüber Rogers, der neutraler formuliert: Exulibus constat Belgis, pietatis amore / Externum e patria qui subiere solum. Vignau-Wilberg (Anm. 41), S. 52. 
Sed sincera fides, hominum nullo illita fuco.

$[\ldots]^{43}$

[Verachte nicht die kleinen Häuser, du siehst, dass für uns neue Mauern aus kleinen Anfängen erbaut wurden. Zuvor wurde dieser Ort von Kutten tragenden Wüstlingen bewohnt und von der unreinen Herde besudelt. Aber den Betrug, der sich unter weißer Kleidung verbarg, erkannte dein Großvater, dem schurkische Taten verhasst waren. Er stellte den heiligen Kult wieder her und entfernte die träge Herde aus ihrem alten Sitz, was hier nur kurz erwähnt sein soll. Das grausame Stürmen des Papstes verschlug Männer, die inzwischen aus ihrer Heimat vertrieben worden waren, in dieses Gebiet. Für alle durch die Unbill Erschöpften, die Mangel an allem litten, war nicht Verbrechen oder Frevel Grund des Exils, sondern ihre aufrichtige Frömmigkeit, von keiner menschlichen Verstellung verdorben. [...] ]

Als Vergleich bietet sich hier die 14 Jahre ältere Beschreibung der Stadt Frankenthal durch Rogers an. Weit ausgreifend zeichnet der englische Dichter-Diplomat vor dem Auge des Lesers ein lebendiges Bild der Stadt mit all ihren statischen Elementen (wie etwa dem Rheinkanal, der ihm offenbar besonders erwähnenswert schien)..$^{44}$ An so einem detaillierten Panorama der örtlichen Verhältnisse ist Mirou anscheinend nicht sonderlich interessiert. Er verweist im Anschluss an die oben zitierte Passage, und bevor er die wirtschaftliche Potenz der jungen Stadt lobt, lediglich auf die Mehrsprachigkeit der Frankenthaler Gemeinden, indem er die Stadt von sich selbst sagen lässt:

Vrbibus aduecti variis, \& dispare lingua,

Mente, animo, cultu, me statuere pares

[Aus verschiedenen Städten zugezogen, von der Sprache her verschieden, aber gleich in Geist, Herz und Glauben haben sie mich erbaut. $]^{45}$

Die Betonung der wirtschaftlichen Bedeutung, der Bienenfleiß ihrer Bewohner, ist für Mirous Anliegen, fortgesetzte fürstliche Protektion und Förderung zu erhalten, zentral. Ansonsten nutzt er sein Stadtlob für konfessionelle Polemik. Der Frevel der durch ihre weiße Kutte kenntlich gemachten Augustiner Chorherren liegt dabei nur implizit in ihrem Katholizismus. Tatsächlich prangert er in erster Linie Verhaltensweisen an, die auch innerhalb ihres eigenen Wertekanons anstößig wären, so vor allem luxuria, die Geilheit (cinaedis) und acedia, die Faulheit (pecus iners) neben den unspezifischen fraudes. Ihre Anrechte verloren sie

43 Mirou: Threnodia (Anm. 19), S. 9 f. Ndr.: Gamer (Anm. 2), S. 267 f., V. 120-132.

44 Scilicet in Rhenum fossam deduxit ab urbe, Vignau-Wilberg (Anm. 41), S. 52.

45 Mirou: Threnodia (Anm. 20), S. 19. Ndr.: Gamer (Anm. 2), S. 268, V. 128. 
aber nach Mirou auch weil sie den ihnen anvertrauten Ort verwahrlosen ließen, ein wiederum mehr reformierter Gedankengang:

Iam vocor urbs, claustrum putridum, \& mapalia quondam

[Schon werde ich Stadt genannt, die ich einst ein morsches Kloster und ein paar Hütten war. $]^{46}$

Mirous Perspektive in diesem Werk ist die eines persönlich Betroffenen, und so drückt er in seiner Stadtbeschreibung einen Antipapismus aus, wie er sich ähnlich auch in der zuvor genannten Trauerdichtung an Casimir bei verschiedenen Autoren findet. Natürlich werden Antipapismus und Antikatholizismus in den Trauergedichten auf den Administrator in den Kontext der Lebensleistung Casimirs gesetzt und die Trauerdichtungen der anderen Kurpfälzer präsentieren ihn als Held und Beschützer der Reformierten:

Ille Isræliticus terra committitur Hector

Qui Satanæ terror, papæ, \& Vbique fuit.

[Jener der auserwählten Gottes Hector wird der Erde übergeben, der überall ein Schrecken für Satan und Papst war.] $]^{47}$

um dessen Standarte sich die Rechtgläubigen sammeln konnten:

Sicut enim partes miles distractus in omnes

Ad numerum refluit cùm su signa videt:

Christi militibus sic cedere fortè coactis

Ad rabiem mundi Pontificumque minas.

[So wie der versprengte Soldat zu seiner Pflicht zurückkehrte, nachdem er sein Feldzeichen sah: Da die Streiter Christi auf diese Weise ermutigt wurden, der Tollheit der Welt und den Drohungen der Priester entgegenzutreten. $]^{48}$

Für diese Sichtweise gibt es im hier herangezogenen Vergleichskorpus weitere Belegstellen. Für Mirou aber waren diese Elemente Bestandteil einer eigenen

46 Mirou: Threnodia (Anm. 20), S. 19. Ndr.: Gamer (Anm. 2), S. 268, V. 142. Entsprechendes findet sich bereits bei Rogers, aber nicht so scharf formuliert: Cernite, quis credat lustris coijsse duobus / Tot profugos, structos tot profugisque lares. / Ante sacer monachis tantum et sine nomine pagus, / Sorduit excultae forma nec urbis erat. Vignau-Wilberg (Anm. 42), S. 52.

47 Kahl (Anm. 24): Elegiacum, S. 6.

48 M. Mattheus Trogerus: Elegia in obitum illustrissimi ducis Iohannis Casimiri. In: Carmina Funebria (Anm. 22), S. 17. 
Lebensbeschreibung, die sich in den Kontext seiner fragmentarischen Biographie stellen lassen.

Konfessionelle Positionierung und Propaganda ist in der Dichtung, die den reformierten Positionen nahesteht, nicht ungewöhnlich. Die Polemik kann dabei sehr aggressiv werden, wie beispielsweise in den Lacrumae Haidelbergenses von 1624, einem Nachruf auf die reformierte Kurpfalz. ${ }^{49}$ Die Perspektive von 1592 war natürlich noch eine andere. Es galt, die gewünschte Politik optimistisch mitzugestalten. Bei dieser propagandistischen Einflussnahme zeigt sich Henric Mirou im Vergleich zu den Kurpfälzer Intellektuellen als einer der schärfer polemisierenden Dichter. Bei all dem erhofften Eigennutzen ist er in seinen beiden Gedichten dennoch auch Fürsprecher einer ganzen Gruppe, die der niederländischen Exulanten. Seine persönlichen, aber auch die kollektiven Erfahrungen seiner Gemeinde ließen die konfessionsbezogene Verse bei ihm nicht als Beiwerk im Hintergrund werden, sondern wesentliches Element und Anliegen. So bieten seine Threnodia und das Carmen Gratulatorium einen besonders lebendigen Eindruck von dieser durch konfessionelle Spannungen und Umbrüchen geprägten Phase der kurpfälzischen Geschichte.

49 Hermann Wiegand: Lacrumae Haidelbergenses. Eine neulateinische epische Klage über die Eroberung Heidelbergs durch Tilly 1622. In: Wiegand (Anm. 5), S. 127-166. 